\title{
CLOUD-BASED MULTI-TENANCY MODEL FOR SELF-SERVICE PORTAL
}

\author{
Jitendra Maan ${ }^{1}$ and Niranjan Mantha ${ }^{2}$ \\ Tata Consultancy Services Limited, India \\ jitendra.maan@tcs.com \\ niranjan.manthaetcs.com
}

\begin{abstract}
A multi-tenant portal implementation extends the capabilities of an enterprise by enabling several customers to run independently on the same portal infrastructure hosted by a service provider. However, building multi-tenant solutions requires addressing several technical challenges, but service providers/solution developers can build and deploy scalable, customizable, manageable, and cost-effective multi-tenant solutions. Addressing multi-tenancy is a key consideration in implementing Cloud enabled enterprise portal solutions which are becoming an international phenomenon, driven by both local demand as well as global reach by medium or large enterprises that are in need of reducing cost of infrastructure/ hosting.

The paper enlighten the focus on the core of multi-tenant portal infrastructure along with key design elements that need to be considered in providing Cloud-enabled multi-tenant portal solutions. The paper would not focus on any specific vendor or their product suites but it acts as a synopsis for developer and architect community to strategize their thinking towards right portal architecture by gaining insights on multi-tenant portal features, key benefits and portal landscape.
\end{abstract}

\section{KEYWORDS}

Multi-tenancy, Portal Infrastructure, Cloud-based Model, Enterprise Portal, Self-service Portal

\section{INTRODUCTION}

Cost savings and increase in revenue are always the key business challenge to face in today's competitive market place. However, increase in revenue is directly impacted by various factors like -continuously adding new customers, retain customer by offering new innovative services. Each enterprise, Medium or large, needed an enterprise-proven on-Demand business portal solution fulfilling the demand of their employees, customers and partners.

The very basic goal of multi-tenant portal infrastructure is to improve productivity, enhance communication, collaboration and performance by shortening the decision cycles at different levels and also to bring people, resources and processes together to create an exponential economic value to the customer. Today, Software service providers are continuously challenged in architecting standards based portal solutions that can manage lots of customers on a single code base but in a more coordinated way than the standard multi-site installation.

The most critical features when evaluating or considering multi-tenant enterprise portals include a cost-effective deployment, a secure solution, an enterprise proven system (scalability) and the ability to integrate with all major technology platforms. However, multi-tenancy is ultimately

Sundarapandian et al. (Eds) : CCSEA, EMSA, DKMP, CLOUD, SEA - 2014

pp. 103-110, 2014. (C) CS \& IT-CSCP 2014

DOI : $10.5121 /$ csit. 2014.4310 
about cost efficiency because its goal is to reduce the number of instances, and to align everyone's schemas, it becomes cheaper and easier to manage. Basically, different levels of multitenancy are just different trade-off points on the cost curve.

Customer specific branding and corporate identify are clearly the winning streak for multitenant portal infrastructure. Multi-tenant portal even bring more flexibility by allowing administrators (both Global and Tenant) to divide a portal into logical partitions, each with its own parallel entry points and dedicated content and navigation for exclusive use of each customer. A shared portal infrastructure (multi-tenant portal) unifies portal administration in a single interface and simplifies management of customer intranets, websites and self-service customer portals.

\section{OVERVIEW OF MUlTi-TenANT SElF SERVICE PORTAL}

Multi-tenant Portal is a single physical portal divided into several logical Portals. A multitenant portal environment allows parallel entry points into the portal, and each entry point is referenced by a unique URL that is assigned to the end User.

In a multi-tenant portal environment, each customer instance is known as Portal Tenant. Each portal tenant comprises a set of secure portal objects and set of services that are highly customized for the respective customer, business users, administrators and the service provider. In addition, the content presented to business users through a tenant is customized and branded by designers to suit the corporate identity of the customer. Within a single portal infrastructure, enterprise can provide content which is unique to different user groups and apply access controls and permissions to customized content on a per user, group, or role basis. There are basically two types of administrator roles in a multi-tenant portal infrastructure -

- Tenant administrator and

- Global administrator

Tenant administrator is a specialized tenant user who manages tenant specific users or content in the design time environment of a multitenant portal. Global administrator is one who has access to cross tenant users or content and this role is normally defined by super user of the portal. The core of the multi-tenant portal infrastructure is shown in the diagram below:-

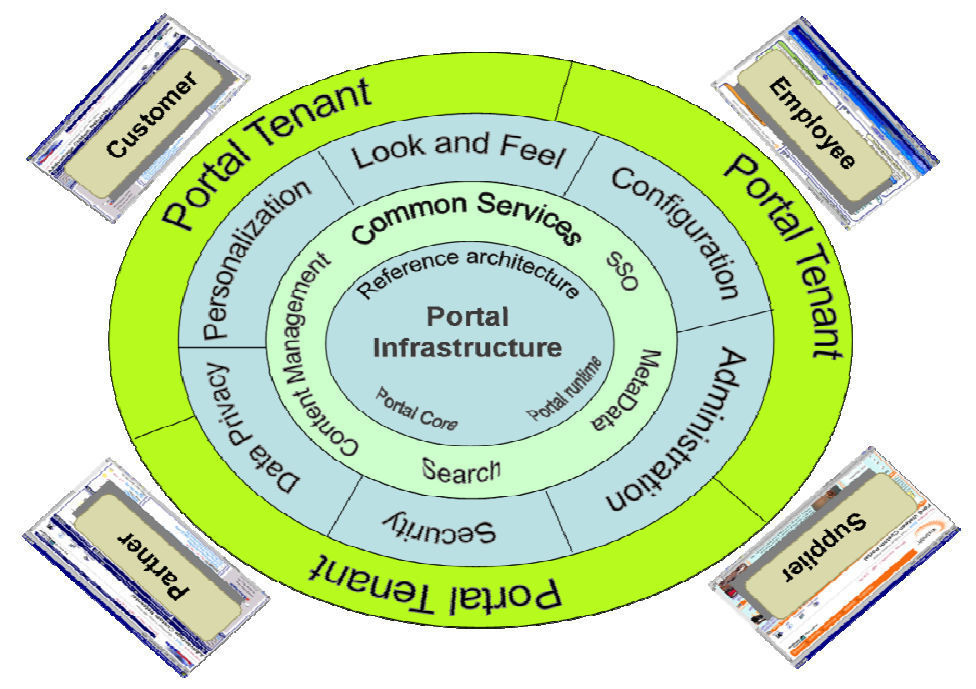

Fig.1. Multi-tenant Portal Infrastructure 


\section{Key DESign CONSIDERATIONS IN MUlti-Tenant Portal}

Multi-tenancy efficient architecture is an important requirement for Web-delivered solutions as well as enterprise proven on-Demand business portals accessed by customers, employees and trade partners.

In case of multi-tenancy, single instance of the portal serves multiple tenants (i.e. Client organizations) but in case of multi-instance architecture, separate portal instances e.g. Virtual portals are setup for different client organizations. With a multi-tenant architecture, a portal application is designed to virtually partition its data and configuration so that each client organization works with a customized virtual portal instance. The multi-tenancy support is the key decision in designing enterprise portal solutions.

The below points describe the key design elements that need to be considered for Cloud enabled multi-tenant Portal.

- Addressing variations in tenant requirements -

- User Interface/Look and feel

- Workflows

- Service orchestration and implementation

- Entities and Database extensions

- Authentication and Authorization

- Customization i.e. One time setup requirement for different customers

- Degree of Configurability

- Collect, Collate \& Configure meta-data at each level

- Data security and Isolation

- Fine grained role based access

- Unique user experience through personalized look and feel

- Tenant provisioning through provisioning Virtual portal instance

- Keeping Customer/vendor data separated and secured

- SOA based architecture \& Web services based messaging/event model for middle-tier

- Data center economics

A typical portal implementation offers a lot of features (out of box or available on customization) which can be leveraged by service providers to provide a customized look and feel in offering branding services to their customers. Another advantage of taking portal solution path is the rich out-of-box feature support based on open standards and portlets, the power engine for Portal framework are modeled against portlet specification standards like JSR 168 or JSR 286. Each portlet can be made configurable for each service vendor or customer based on their needs.

\subsection{Virtual Portal Implementation - Clone and Configure Approach}

Most of the open source/traditional portal vendors provide a clone-and-configure approach to the implementation \& deployment of the portal for multi-tenant applications i.e. the ability to clone a base portal and configure it as per end-user/vendor/partner needs. Several Open Source portal vendors support the concept of a virtual portal which is basically a logical copy of an existing base portal utilizing the same hardware and software resources.

\subsubsection{Degree of Configurability and Customization}

To enable a high level of reuse, the degree of configurability in the portlet design is achieved by supporting customer and/or vendor-specific settings through name and-value pair configurations 
such as Customer IDs or customer's service endpoints, to offer each customer specific look-andfeel thereby offering a highly scalable, configurable and multi-tenant efficient solutions.

Following are the recommendations for portal environment based multi-tenant applications:

- Each portlet must be configurable for each customer, since a service provider shares portlets across individual vendors.

- To enable a high level of reuse, the degree of configurability in portlets must support subscriber or vendor specific settings, such as vendor's service endpoints, to provide vendor or subscriber-specific look-and-feel, with specific settings to indicate which form fields or action buttons should be rendered to end-users/administrators based on role based access.

In addition to configuration at the customer level, the individual vendors/channel partners must be able to perform the configuration without the support from the customer or service providers.

\subsubsection{Security Isolation}

Each virtual portal supports isolation of user populations for each tenant through a multi-tenant LDAP tree structure in a single instance of a directory Server. Through dynamic profiles, an application developer can define variables in a common 'profile set' that can be configured by each tenant's administrator at runtime thereby, enabling the unique branding for different tenants. Tenant specific customizations are created by applying those configured profiles to their portlets at runtime to change the appearance, content and behavior of the portlet.A sample scenario as outlined below:

- Create a multi-tenant user directory structure in LDAP by:

- Create a realm for each vendor or customer i.e. a separate tree hierarchy starting at $\mathrm{dn}$ [dc=vendor $1, \mathrm{dc}=\mathrm{com}]$

- And, for each security realm, a security context entry must be created, configured and mapped to user population of each service provider or end customer organization.

- Collaboration and Knowledge Management

- Extended Collaboration

- Social Business Extensions

\subsubsection{Unique User Experience}

Rich and unique user experience, at very high level, consists of:

- Look and feel for users or groups (belonging to either customer or end user organization) and

- Structure and scope of user role

Most portal vendors provide out-of-box features which can be utilized and customized in a unique manner to create a distinct look and feel that will go consistent with the customer branding needs. Also it provides flexibility to the vendors to customize the look and feel based on their specific business requirements to serve their end-customer effectively and efficiently.

\section{MUlti-Tenant PoRTAl IMPlementation}

A multi-tenant portal extends the capabilities of self-service portal by enabling a single service provider to offer granular services based on SAP technology and applications to multiple customers from a single portal infrastructure.

The service provider partitions a single portal installation into several logical units. Each partition is a portal with its own dedicated content and navigation for the exclusive use of a customer. The 
term tenant is used to describe each logical partition. The service provider sets up parallel entry points into the portal-one entry point per tenant. Each entry point is referenced by a unique URL gateway, also referred to as a portal alias. The users of a tenant can access only the information of their tenant.

The following figure shows a layered multi-tenant portal architecture with three tenants:

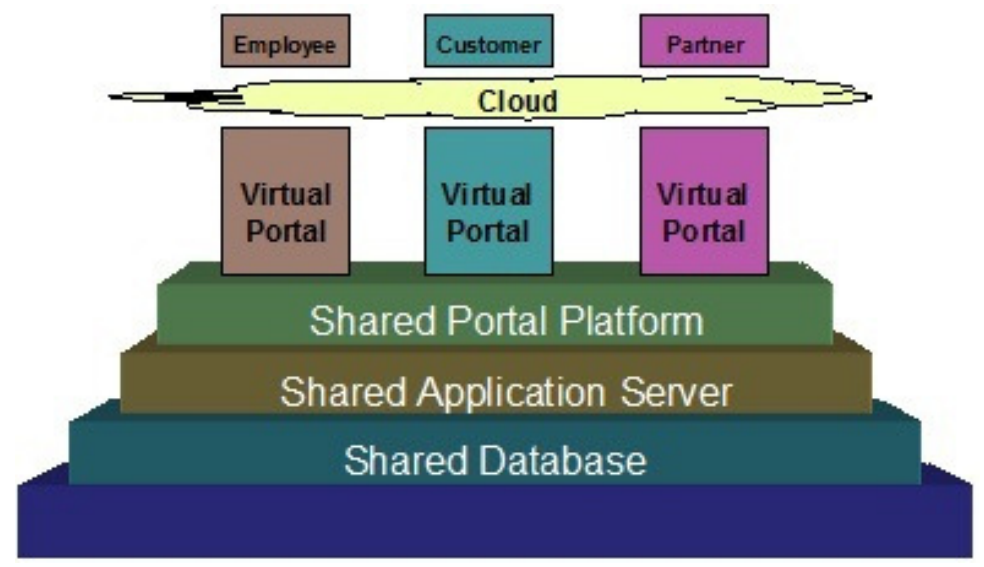

Fig.1. Layered Multi-tenant Portal Architecture

The overall strategy for enterprise portal implementation should consider the following fundamental requirements for multi-tenant efficient applications:

- Describes the multi-tenant portal environment

- Explains how to configure a single portal landscape to support multiple tenants

- Outlines how to secure the multi-tenant portal environment to make sure that content belonging to one enterprise is not delivered to members of other enterprises.

- Discusses administration tasks, such as initial preparation, configuration, and daily maintenance

- Details how to manage the customized portal content of individual enterprises within a single portal landscape

- Details how to manage the users in a multi-tenant portal environment

- Details how to customize the look and feel of each portal tenant to incorporate the customers' corporate identity

\section{KEY FEATURES SUPPORTED In MULTI-TENANT PORTAL}

The following features support the multi-tenant portal environment:

- Customer-specific branding and corporate identity

- Per user/group/role permission infrastructure for controlling access to portal content

- Delegated administration for setting up different customized content

- Mass customization and configuration operations

- User management filtering mechanism for controlling access to users, groups, and roles per tenant

- Multi-language user interface support 
Within a single portal infrastructure, the service provider can:-

- Provide content which is unique to different user groups

- Isolate the content of a group

- Apply access controls and permissions to customized content on a per user, group, or role basis

\section{Multi-Tenant Portal Landscape}

The following figure provides an example of a multi-tenant portal system landscape:

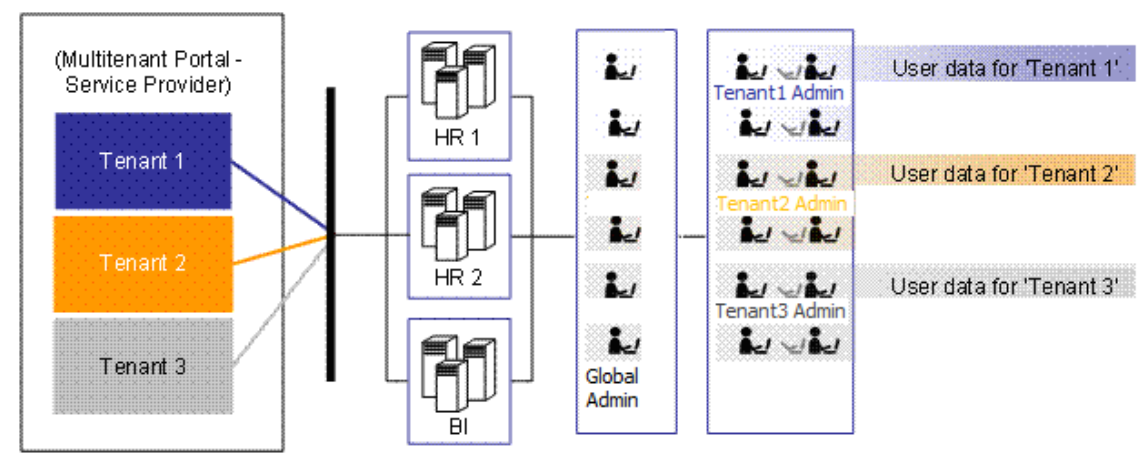

Fig.3. Multi-tenant Portal Landscape

The Portal system landscape in the figure above comprises two Human Resources (HR) systems, one Business Intelligence (BI) system, and a central user administration, which are all connected to the multi-tenant portal. These systems present information to the following tenants: Tenant 1 , Tenant 2, and Tenant 3 .

Users of the different tenants are defined in separate clients either in one or several systems. For example, when a user from Tenant 1 makes a request in the portal, that user is authenticated in the central user administration, which also checks the authorization level of the user for the requested data.

After the request is authenticated and the user has permission for the requested data, the information is presented in the portal based on branding and corporate identity of respective Tenant.

\section{KEY TAKEAWAYS}

There are several benefits of using a multi-tenant portal landscape. Some of the key takeaways as below: -

- Single portal infrastructure would deliver services to multiple customers. There is a definite cost reduction through lower ongoing total costs for operating a multitenant environment rather than multiple portals.

- Global administrators provide centralized control by defining various tenants that are hosted in the portal infrastructure. Besides this, administrator defines the portal alias, the path to the company logo as well as other tenant specific information.

- System landscape is less complex; thereby reducing overall risk to businesses. It also helps to achieve system landscape optimization.

- Standardization and best practices across single portal infrastructure improves the quality standards. 
- The cost for the service and the cost per user is drastically reduced by standardization and reuse of services by different portal tenants.

- Each tenant specific configuration becomes easy through metadata associated with each tenant. It does not require making changes in codebase each time a new portal tenant is instantiated.

However, besides above benefits, multi-tenant portal solutions can also be used to structure the portal for different business units, as well as subsidiaries within the same organization. Several service providers leverages its rich experience in providing Portal solution offerings across its global customer base to help companies achieve their objective of a state-of-the-art self-service multi-tenant portal.

\section{CONCLUSION}

The multi-tenancy is the most significant paradigm shifts in portal application design extensively dependent on metadata at each level to configure the way the application appear/behave for the users. However, the multitenant enterprise portal is a vital component to businesses in reducing the cost and also empowers customer to identify the solutions to their problems quickly.

From my experience, I strongly recommend multi-tenant architecture when creating enterprise portal solutions as it allows vendors to provide self-service capabilities to customers and prospects for key marketing, sales and support activities and branding services for their partners/channels.

In summary, the multi-tenant architecture is an important design decision when working with potential business solutions that have similar core functionality but fundamentally differ in various aspects such as UI, Layout and workflows. The basic concepts explained here are the foundations for the Multi-Tenant Architecture. Depending on your organization needs, there may be extra steps to utilize the design in an effective manner.

\section{REFERENCES}

[1] Stefan Fenz, "Increasing knowledge capturing efficiency by enterprise portals" VINE,Vol. 42 Iss: 2, pp. $237-250$

[2] Paul Hawking, Andrew Stein, "B2E Portal Maturity: An Employee Self-Service Case Study."School of Information Systems, Faculty of Business, Victoria University, (2004)

[3] Link, D. (2001), "How HR Can Shape Corporate Portals", HRMagazine, 46(9), 131-137.

[4] Xuxu, Z., Qingzhong, L., \&Lanju, K. (2010). A data storage architecture supporting multi-level custo-mization for saas. In Web information systems and applications conference (wisa), 2010, 7th (pp. $106\{109)$.

[5] Shilakes, C. \&Tylman, J. (1998), "Enterprise Information Portals", Merril Lynch report, Located at http://emarkets.grm.hia.no/gem/topic7/eip_ind.pdf Accessed March 2003.

[6] Wiscombe, J. (2001), "Using Technology To Cut Costs", Workforce, September 2001, Located at http://www.workforce.com/archive/feature/22/29/82/index.php Accessed August 2002.

[7] Killen and Associates (2000), "Communicating with Employees Via Digital Delivery Systems", Report 2000.

[8] Yu, D., Wang, J., Hu, B., Liu, J., Zhang, X., He, K., \& Zhang, L. (2011). A practical architecture of cloudification of legacy applications. In Services (services), 2011 ieee world congress on (pp. 17\{24).

[9] Hamerman, P. (2002), "Extending Employee Relationships with Web Applications," Presentation to SAPPHIRE Lisbon Conference, July 2002.

[10] Brosche, C. (2002), "Designing the Corporate Portal", Masters Thesis, Department of Computer Science, University of Gothenburg, Sweden, May.

[11] Barron M. Cited at Internet Retailer, (2002), "Retail web-based self-serve isn't just for customers,it's for employees", Located at http://www.internetretailer. com/dailynews.asp?id=6688 Accessed September 2002 . 
[12] Zhang, Y., Wang, Z.,Gao,B.,Guo, C., Sun, W., \& Li, X. (2010). An elective heuristic for on-line tenant placement problem in SAAS. In Web services (icws),2010ieeeconference on (pp. 425-432).

\section{AUTHORS}

Jitendra Maan, a versatile IT Professional with a total of more than 17 years of experience spread across various domains in IT Industry and he is currently working with Tata Consultancy Services Limited in a leading role to drive Social Computing and Java and Open Source Solutions and Offerings to address customer needs in HiTech ISU. Jitendra practices technology consulting, enterprise architecture and evangelizes social computing initiatives within TCS and has successfully delivered technology solutions for globally distributed clientele. Jitendra is certified in Project Management (CIPM) by Project Management Associates (PMA)India and has successfully achieved

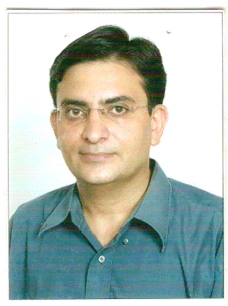
the standards of TOGAF 8 Certification program. Jitendra has a proven track record of sharing technology thought leadership in various international conferences and also presented his research work in various international events/forums. Jitendra is also a member to professional bodies like PMA (Project Management Associates), IEEE (Institute of Electrical and Electronics Engineers, Computer Society of India (CSI) Delhi Chapter, Open Group AEA Delhi Chapter.

NiranjanMantha, having 15 years of IT experience across different geographies. He is currently managing the Java and Open Source opportunities and initiatives in HiTech ISU. Niranjan is a TOGAF 9 Certified Practitioner an Certified SCRUM Master, having vast knowledgeable in the area of Amazon Cloud Services. 